\title{
Estimates on the Transition Densities of Girsanov Transforms of Symmetric Stable Processes
}

\author{
Renming Song ${ }^{1}$
}

Received September 16, 2004; revised November 15, 2004

In this paper, we first study a purely discontinuous Girsanov transform which is more general than that studied in Chen and Song [(2003), J. Funct. Anal. 201, 262-281]. Then we show that the transition density of any purely discontinuous Girsanov transform of a symmetric stable process is comparable to the transition density of the symmetric stable process. The same is true for the Girsanov transform introduced in Chen and Zhang [(2002), Ann. Inst. Henri poincaré 38, 475-505]. As an application of these results, we show that the Green function of Feynman-Kac type transforms of symmetric stable processes by continuous additive functionals of zero energy, when exists, is comparable to that of the symmetric stable process.

KEY WORDS: Transition density; Green function; Girsanov transform; Dirichlet forms; symmetric Markov processes; martingale additive functionals; additive functionals of zero energy; Feynman-Kac semigroups; symmetric stable processes; Brownian motion.

AMS 2000 MATHEMATICS SUBJECT CLASSIFICATION: Primary 60J45; 60J40; Secondary 35J10; 47J20.

\section{INTRODUCTION}

Suppose that $\left(X, \mathbb{P}_{x}\right)$ is a Brownian motion in $\mathbb{R}^{d}$ and let $\mathcal{M}_{t}$ be the $\sigma$-field generated by $\left\{X_{s}, s \leqslant t\right\}$. When $b$ is an $\mathbb{R}^{d}$-valued function on $\mathbb{R}^{d}$ satisfying certain natural conditions,

$$
L_{t}=\exp \left(\int_{0}^{t} b\left(X_{s}\right) d X_{s}-\frac{1}{2} \int_{0}^{t}\left|b\left(X_{s}\right)\right|^{2} d s\right)
$$

\footnotetext{
${ }^{1}$ Department of Mathematics, University of Illinois, Urbana, IL 61801, USA.

e-mail: rsong@math.uiuc.edu
} 
is a nonnegative local martingale under each $\mathbb{P}_{x}$ and thus is a supermartingale multiplicative functional of $X . L_{t}$ defines a family of probability measure $\left\{\tilde{\mathbb{P}}_{x}: x \in \mathbb{R}^{d}\right\}$ on $\mathcal{M}_{\infty}$ by $d \tilde{\mathbb{P}}_{x}=L_{t} d \mathbb{P}_{x}$ on $\mathcal{M}_{t}$. Let $\tilde{X}_{t}$ be the process $X_{t}$ under this family of measures $\left\{\tilde{\mathbb{P}}_{x}: x \in \mathbb{R}^{d}\right\}$. The process $\tilde{X}$ is called a Girsanov transform of $X$. It is well known that, when $b$ satisfies certain natural conditions, the transition density of $\tilde{X}$ has Gaussian lower and upper estimates. The main purpose of this paper is to study upper and lower estimates on the transition densities of Girsanov transforms of symmetric stable processes.

We are mainly concerned with two types of Girsanov transforms of the symmetric stable process: one is a purely discontinuous Girsanov transform which is a generalization of the purely discontinuous Girsanov introduced in Ref. 8, the other is the Girsanov transform studied in Ref. 9.

Let's first briefly recall the purely discontinuous Girsanov transform studied in Ref. 9. Let $E$ be a Lusin space, $\mathcal{B}(E)$ the Borel $\sigma$-field on $E$, and $m$ a $\sigma$-finite measure on $\mathcal{B}(E)$ with $\operatorname{supp}[m]=E$. Let $\left\{X, \mathbb{P}_{x}, x \in E\right\}$ be an $m$-symmetric, transient Borel standard process on $E$. Adjoined to the state space $E$ is an isolated point $\partial \notin E$; the process retires to $\partial$ at its lifetime $\zeta=\inf \left\{t: X_{t}=\partial\right\}$. We will use $E_{\partial}$ to denote $E \cup\{\partial\}$. Suppose that the semigroup $P_{t}$ of $X$ has a transition density $p(t, x, y)$ for each $t>0$. Let $(N, H)$ be a Lévy system for $X$ (cf. Ref. 1 and Theorem 47.10 of Ref. 17); that is, $N(x, d y)$ is a kernel on $(E, \mathcal{B}(E))$ and $H_{t}$ is a positive continuous additive functional of $X$ with bounded 1-potential such that for any nonnegative Borel function $f$ on $E \times E$ that vanishes on the diagonal and is extended to be zero off $E \times E$,

$$
\mathbb{E}_{x}\left(\sum_{s \leqslant t} f\left(X_{s-}, X_{s}\right)\right)=\mathbb{E}_{x}\left(\int_{0}^{t} \int_{E} f\left(X_{s}, y\right) N\left(X_{s}, d y\right) d H_{s}\right)
$$

for every $x \in E$.

Let $F$ be a bounded function on $E \times E$ that vanishes on the diagonal, we extend $F$ to $E_{\partial} \times E_{\partial}$ by setting it to be zero off $E \times E$. Recall (see Ref. 7) that a bounded function $F$ on $E \times E$ vanishing on the diagonal is said to be in the class $\mathbf{J}(X)$ if

$$
\limsup _{t \downarrow 0} \mathbb{E}_{x \in E}\left(\int_{0}^{t} \int_{E}|F|\left(X_{s}, y\right) N\left(X_{s}, d y\right) d H_{s}\right)=0 .
$$

If $F \in \mathbf{J}(X)$ also satisfies the condition $\inf _{x, y \in E} F(x, y)>-1$, then the process 


$$
t \mapsto \sum_{0<s \leqslant t} F\left(X_{s-}, X_{s}\right)-\int_{0}^{t} \int_{E} F\left(X_{s}, y\right) N\left(X_{s}, d y\right) d H_{S}
$$

is a martingale and its Doleans-Dade exponential is (cf. Theorem 9.39 of Ref. 15)

$$
\begin{aligned}
L_{t} & =\exp \left(-\int_{0}^{t} \int_{E} F\left(X_{s}, y\right) N\left(X_{s}, d y\right) d H_{s}\right) \prod_{s \leqslant t}\left(1+F\left(X_{s-}, X_{s}\right)\right) \\
& =\exp \left(\sum_{s \leqslant t} \ln \left(1+F\left(X_{s-}, X_{s}\right)\right)-\int_{0}^{t} \int_{E} F\left(X_{s}, y\right) N\left(X_{s}, d y\right) d H_{s}\right) .
\end{aligned}
$$

$L_{t}$ is a nonnegative local martingale. The local martingale $L_{t}$ is obviously a nonnegative supermartingale multiplicative functional of $X$. Therefore by Section 62 of Ref. 17, $L_{t}$ defines a family of probability measure $\left\{\tilde{\mathbb{P}}_{x}: x \in\right.$ $E\}$ on $\mathcal{M}_{\infty}$ by $d \tilde{\mathbb{P}}_{x}=L_{t} 1_{\{t<\zeta\}} d \mathbb{P}_{x}$ on $\mathcal{M}_{t}$. Let $\tilde{X}_{t}$ be the process $X_{t}$ under this family of measures $\left\{\tilde{\mathbb{P}}_{x}: x \in E\right\}$. The process $\tilde{X}$ is called a purely discontinuous Girsanov transform of $X$. Under the further assumption that $F \in \mathbf{A}_{2}(X)$ (see Ref. 8 for the definition) it was shown that the Green function of $\tilde{X}$ is comparable to that of $X$. The last result is a generalization of the results in Ref. 10 to discontinuous processes.

In Section 2 of this paper, we will first introduce a class of purely discontinuous Girsanov transforms more general than that introduced above and then, by using the recent result of Ref. 4, we show that, in the special case when $X$ is a symmetric stable process in $\mathbb{R}^{d}$, the transition density of a purely discontinuous Girsanov transform $\tilde{X}$ of $X$ is comparable to that of $X$.

In Section 3, we study the Girsanov transform introduced in Ref. 9. We show that the transition density of this type of Girsanov transform of a symmetric stable process $X$ is comparable to that of $X$. As an application of this result, we show that the Green function, when exists, of Feynman-Kac type transforms of symmetric stable processes by continuous additive functionals of zero energy, is comparable to that of the symmetric stable process.

\section{PURE JUMP GIRSANOV TRANSFORMS}

Let $E$ be a Lusin space, $\mathcal{B}(E)$ the Borel $\sigma$-field on $E$, and $m$ a $\sigma$-finite measure on $\mathcal{B}(E)$ with $\operatorname{supp}(m)=E$. Suppose that $X=(\Omega, \mathcal{M}$, $\left.\mathcal{M}_{t}, \theta_{t}, X_{t}, \mathbb{P}_{x}\right)$ is an $m$-symmetric right Markov process on $E$. More precisely, the right continuous process $[0, \infty) \ni t \mapsto X_{t}$ is defined on the sample space $(\Omega, \mathcal{M})$, adapted to the filtration $\left\{\mathcal{M}_{t}\right\}$, and under $\mathbb{P}_{x}$ is a strong 
Markov process with initial condition $X_{0}=x$. The shift operators $\theta_{t}, t \geqslant 0$, satisfy $X_{s} \circ \theta_{t}=X_{s+t}$ identically for $s, t \geqslant 0$. Adjoined to the state space $E$ is an isolated point $\partial \notin E$; the process $X$ retires to $\partial$ at its "lifetime" $\zeta=\inf \left\{t: X_{t}=\partial\right\}$. The transition operators $P_{t}, t \geqslant 0$, are defined by

$$
P_{t} f(x):=\mathbb{E}_{x}\left[f\left(X_{t}\right)\right]=\mathbb{E}_{x}\left[f\left(X_{t}\right) ; t<\zeta\right]
$$

(Here and in the sequel we use the convention that a function defined on $E$ takes the value 0 at the cemetery point $\partial$.) $P_{t}$ may be viewed as operators on $L^{2}(E, m)$; as such they form a strongly continuous semigroup of selfadjoint contractions. The Dirichlet form associated with $X$ is the bilinear form

$$
\mathcal{E}(u, v):=\lim _{t \downarrow 0} \frac{1}{t}\left(u, v-P_{t} v\right)_{m}
$$

defined on the space

$$
\mathcal{F}:=\left\{u \in L^{2}(E, m): \sup _{t>0} \frac{1}{t}\left(u, u-P_{t} u\right)_{m}<\infty\right\} .
$$

It is known that the Dirichlet form $(\mathcal{E}, \mathcal{F})$ is quasi-regular. We know (see, for instance, Ref. 6) that a Dirichlet form is quasi-regular if and only if it is quasi-homeomorphic to a regular Dirichlet form on a locally compact separable metric space. Thus without loss of generality, we assume throughout this section that $E$ is a locally compact separable metric space and that $(\mathcal{E}, \mathcal{F})$ is a regular Dirichlet form. Let $\mathcal{F}_{b}=\mathcal{F} \cap L^{\infty}(E, m)$, and let $\mathcal{F}_{e}$ be the family of $\mathcal{B}(E)$-measurable functions $u$ on $E$ that are finite $m$ a. e. and there is an $\mathcal{E}$-Cauchy sequence $\left\{u_{n}\right\} \subset \mathcal{F}$ such that $\lim _{n \rightarrow \infty} u_{n}=u$ $m$-a. e. on $E$. $\left(\mathcal{E}, \mathcal{F}_{e}\right)$ is called the extended Dirichlet space of $(\mathcal{E}, \mathcal{F})$.

It is well known that for $u \in \mathcal{F}_{e}, u$ has a quasi-continuous version $\tilde{u}$ and $\tilde{u}\left(X_{t}\right)$ has the following Fukushima decomposition

$$
\tilde{u}\left(X_{t}\right)=\tilde{u}\left(X_{0}\right)+M_{t}^{u}+N_{t}^{u}, \quad t \geqslant 0 .
$$

Here $M^{u}$ is a martingale additive functional of $X$ and $N^{u}$ is a continuous additive functional of $X$ having zero energy. Note that in general $N^{u}$ is not a process of finite variation. For some examples of additive functionals of zero energy which are not of finite variation (see, for instance, Ref. 9).

Let $\left(N(x, d y), H_{t}\right)$ be a Lévy system for $X$. If we use $\mu_{H}$ to denote the Revuz measure of the positive continuous additive functional $H$, then the jumping measure $J$ and killing measure $\kappa$ of $X$ are given by

$$
J(d x, d y)=\frac{1}{2} N(x, d y) \mu_{H}(d x) \quad \text { and } \quad \kappa(d x)=N(x, \partial) \mu_{H}(d x) .
$$


Furthermore the following Beurling-Deny decomposition holds for $f$, $g \in \mathcal{F}_{e}$,

$$
\begin{aligned}
\mathcal{E}(f, g)= & \mathcal{E}^{(c)}(f, g)+\int_{E \times E}(\tilde{f}(x)-\tilde{f}(y))(\tilde{g}(x)-\tilde{g}(y)) J(d x, d y) \\
& +\int_{E} \tilde{f}(x) \tilde{g}(x) \kappa(d x),
\end{aligned}
$$

where $\mathcal{E}^{(c)}$ is the strongly local part of $\mathcal{E}$. In this section, we assume that $J$ is nontrivial, or equivalently, the process $X$ is discontinuous.

The martingale part $M^{u}$ in (2.1) can be decomposed as

$$
M_{t}^{u}=M_{t}^{u, c}+M_{t}^{u, j}+M_{t}^{u, k},
$$

where

$$
\begin{aligned}
M_{t}^{u, j}= & \lim _{n \rightarrow \infty}\left\{\sum_{0<s \leqslant t}\left(\tilde{u}\left(X_{s}\right)-\tilde{u}\left(X_{s-}\right)\right) 1_{\left\{\tilde{u}\left(X_{s}\right)-\tilde{u}\left(X_{s-}\right) \mid>1 / n\right\}} 1_{\{t<\zeta\}}\right. \\
& \left.-\int_{0}^{t}\left(\int_{\left\{y \in E:\left|\tilde{u}(y)-\tilde{u}\left(X_{s}\right)\right|>1 / n\right\}}\left(\tilde{u}(y)-\tilde{u}\left(X_{s}\right)\right) N\left(X_{s}, d y\right)\right) d H_{s}\right\} \\
M_{t}^{u, k}= & \int_{0}^{t} \tilde{u}\left(X_{s}\right) N\left(X_{s}, \partial\right) d H_{s}-\tilde{u}\left(X_{\zeta-}\right) 1_{\{t \geqslant \zeta\}}
\end{aligned}
$$

and $M_{t}^{u, c}$ are, respectively, the jumping, killing and continuous part of $M^{u}$. The limit in the expression for $M^{u, j}$ is in the sense of convergence in probability and convergence in the norm of the space of square integrable martingales.

Let $\mu_{\langle u\rangle}, \mu_{\langle u\rangle}^{c}, \mu_{\langle u\rangle}^{j}$ and $\mu_{\langle u\rangle}^{k}$ be the Revuz measures associated with the sharp bracket positive continuous additive functionals $\left\langle M^{u}\right\rangle,\left\langle M^{u, c}\right\rangle$, $\left\langle M^{u, j}\right\rangle$ and $\left\langle M^{u, k}\right\rangle$, respectively. Then

$$
\mu_{\langle u\rangle}(d x)=\mu_{\langle u\rangle}^{c}(d x)+\mu_{\langle u\rangle}^{j}(d x)+\mu_{\langle u\rangle}^{k}(d x),
$$

where $\mu_{\langle u\rangle}^{c}$ satisfies $\mu_{\langle u\rangle}^{c}(E)=2 \mathcal{E}^{(c)}(u, u)$,

$$
\mu_{\langle u\rangle}^{j}(d x)=2 \int_{E}(\tilde{u}(x)-\tilde{u}(y))^{2} J(d x d y)
$$

and

$$
\mu_{\langle u\rangle}^{k}(d x)=\tilde{u}(x)^{2} \kappa(d x)
$$


Definition 2.1. Let $F$ be bounded symmetric function on $E \times E$ vanishing on the diagonal, we extend it to $E_{\partial} \times E_{\partial}$ by setting it to be zero off $E \times E . F$ is said to be in the class $\mathbf{I}_{2}(X)$ if for every $x \in E$ and every $t>0$,

$$
\mathbb{E}_{x}\left[\sum_{s \leqslant t} F^{2}\left(X_{s-}, X_{s}\right)\right]=\mathbb{E}_{x}\left[\int_{0}^{t} \int_{E} F^{2}\left(X_{s}, y\right) N\left(X_{s}, d y\right) d H_{s}\right]<\infty .
$$

Since for any bounded $F$ on $E \times E$ vanishing on the diagonal we have

$$
\begin{aligned}
\mathbb{E}_{x}\left[\int_{0}^{t} \int_{E} F^{2}\left(X_{s}, y\right) N\left(X_{s}, d y\right) d H_{s}\right] & \leqslant\|F\|_{\infty} \mathbb{E}_{x} \\
& {\left[\int_{0}^{t} \int_{E}|F|\left(X_{s}, y\right) N\left(X_{s}, d y\right) d H_{s}\right] }
\end{aligned}
$$

for all $x \in E$ and $t>0$, we know that $\mathbf{J}(X) \subset \mathbf{I}_{2}(X)$. From the definition it is easy to see that if $F \in \mathbf{I}_{2}(X)$ satisfies the condition $\inf _{x, y \in E} F(x, y)>-1$, then the function $\ln (1+F)$ is also in $\mathbf{I}_{2}(X)$.

Example. Suppose that $X$ is a symmetric $\alpha$-stable process in $\mathbb{R}^{d}$ for some $\alpha \in(0,2)$. It has a Lévy system $(N, H)$ given by $H_{t}=t$ and $N(x, d y)=\mathcal{A}(d,-\alpha)|x-y|^{-(d+\alpha)} d y$ with

$$
\mathcal{A}(d,-\alpha)=\frac{|\alpha| \Gamma\left(\frac{d+\alpha}{2}\right)}{2^{1-\alpha} \pi^{d / 2} \Gamma\left(1-\frac{\alpha}{2}\right)} .
$$

It is very easy to check that if $F$ is a bounded symmetric function on $\mathbb{R}^{d} \times$ $\mathbb{R}^{d}$ vanishing on the diagonal and satisfying

$$
|F(x, y)| \leqslant C|x-y|^{\beta}, \quad x, y \in \mathbb{R}^{d}
$$

for some $\beta>\alpha / 2$ and $C>0$, then the function

$$
x \mapsto \int_{\mathbb{R}^{d}} \frac{F^{2}(x, y)}{|x-y|^{d+\alpha}} d y
$$

is bounded, and so $F \in \mathbf{I}_{2}(X)$. While on the other hand, when $\alpha<d$, in order to guarantee that $F \in \mathbf{J}(X)$ which is amounts to say (see Refs. 6 and 7) that

$$
\lim _{r \downarrow 0} \sup _{x \in \mathbb{R}^{d}} \int_{B(x, r)} \frac{1}{|x-y|^{d-\alpha}} \int_{\mathbb{R}^{d}} \frac{|F(y, z)|}{|y-z|^{d+\alpha}} d z d y=0,
$$

we need to require that $\beta>\alpha$. 
This example tells us that $\mathbf{I}_{2}(X)$ differs from $\mathbf{J}(X)$ in that the assumption on the behavior of $F$ near the diagonal is weakened.

For any $F \in \mathbf{I}_{2}(X)$ and any positive integer $n$ define

$$
\begin{aligned}
M_{F}^{(n)}(t)= & \sum_{s \leqslant t} F\left(X_{s-}, X_{S}\right) 1_{\left\{\left|X_{s-}-X_{s}\right|>1 / n\right\}} \\
& -\int_{0}^{t} \int_{E} F\left(X_{S}, y\right) 1_{\left\{\left|X_{S}-y\right|>1 / n\right\}} N\left(X_{S}, d y\right) d H_{s} .
\end{aligned}
$$

Then $M_{F}^{(n)}$ is a martingale additive functional of $X$ and for any $x \in E$,

$$
\left\langle M_{F}^{(n)}\right\rangle_{t}=\int_{0}^{t} \int_{E} F^{2}\left(X_{s}, y\right) 1_{\left\{\left|X_{s}-y\right|>1 / n\right\}} N\left(X_{s}, d y\right) d H_{s} .
$$

By the definition of $\mathbf{I}_{2}(X)$ we know that for all $x$ and $t>0$ we have

$$
\int_{0}^{t} \int_{E} F^{2}\left(X_{s}, y\right) N\left(X_{s}, d y\right) d H_{s}<\infty
$$

thus for every $t>0,\left\{\left\langle M_{F}^{(n)}\right\rangle_{t}: n \geqslant 1\right\}$ is a Cauchy sequence in $L^{2}\left(\mathbb{P}_{x}\right)$ for every $x$, and so (see for instance, Ref. 12) the $\operatorname{limit}_{\lim } \lim _{n \rightarrow \infty} M_{F}^{(n)}(t)$ exists in the sense of convergence of the norm of the space of square-integrable martingales and convergence in probability under $\mathbb{P}_{x}$, and the limit is also a martingale additive functional of $X$. We shall use $M_{F}(t)$ to denote this limit. The above argument above shows that the assumption $F \in \mathbf{I}_{2}(X)$ is needed to define the martingale additive functional $M_{F}(t)$ of $X$, which is the starting point of our discussion in this section.

Now we fix an $F \in \mathbf{I}_{2}(X)$ such that $\inf _{x, y \in E} F(x, y)>-1$. Let $L_{F}^{(n)}(t)$ be the solution to

$$
L_{F}^{(n)}(t)=1+\int_{0}^{t} L_{F}^{(n)}(s-) d M_{F}^{(n)}(s)
$$

and $L_{F}(t)$ be the solution to

$$
L_{F}(t)=1+\int_{0}^{t} L_{F}(s-) d M_{F}(s)
$$

It follows from the Doleans-Dade formula that 


$$
\begin{aligned}
& L_{F}^{(n)}(t)=\exp \left(M_{F}^{(n)}(t)\right) \prod_{s \leqslant t} \\
& \left(1+F\left(X_{s-}, X_{s}\right) 1_{\left\{\left|X_{s-}-X_{s}\right|>1 / n\right\}}\right) e^{-F\left(X_{s-}, X_{s}\right) 1_{\left\{\left|X_{s-}-X_{s}\right|>1 / n\right\}}} \\
& =\exp \left(M_{F}^{(n)}(t)+\sum_{s \leqslant t}(\ln (1+F)-F)\left(X_{s-}, X_{s}\right) 1_{\left\{\left|X_{s-}-X_{s}\right|>1 / n\right\}}\right) \\
& =\exp \left(\sum_{s \leqslant t}\left(\ln \left(1+F\left(X_{s-}, X_{S}\right) 1_{\left\{\left|X_{s-}-X_{s}\right|>1 / n\right\}}\right)\right)\right. \\
& \left.\quad-\int_{0}^{t} \int_{E} F\left(X_{S}, y\right) 1_{\left\{\left|X_{s}-y\right|>1 / n\right\}} N\left(X_{S}, d y\right) d H_{S}\right) .
\end{aligned}
$$

and

$$
\begin{aligned}
L_{F}(t) & =\exp \left(M_{F}(t)\right) \prod_{s \leqslant t}\left(1+F\left(X_{s-}, X_{s}\right)\right) e^{-F\left(X_{s-}, X_{s}\right)} \\
& =\exp \left(M_{F}(t)+\sum_{s \leqslant t}(\ln (1+F)-F)\left(X_{s-}, X_{s}\right)\right) .
\end{aligned}
$$

We know that $M_{F}^{(n)}(t)$ converges to $M_{F}(t)$ in probability. Since $F$ is bounded, we know that

$$
|\ln (1+F(x, y))-F(x, y)| \leqslant C F^{2}(x, y), \quad \forall x, y \in E,
$$

thus using the assumption that $F \in \mathbf{I}_{2}(X)$ we can show that

$$
\left.\sum_{s \leqslant t}(\ln (1+F)-F)\left(X_{s-}, X_{s}\right)\right) 1_{\left\{\left|X_{s-}-X_{s}\right|>1 / n\right\}}
$$

converges to

$$
\sum_{s \leqslant t}(\ln (1+F)-F)\left(X_{s-}, X_{s}\right)
$$

in probability. Therefore $L_{F}^{(n)}(t)$ converges to $L_{F}(t)$ in probability. Note that $L_{F}(t)$ is a positive local martingale and thus a supermartingale multiplicative functional of $X$. Therefore by Section 62 of Ref. 17, $L_{F}(t)$ defines a family of probability measures $\left\{\tilde{\mathbb{P}}_{x}, x \in E\right\}$ on $\mathcal{M}_{\infty}$ by $d \tilde{\mathbb{P}}_{x}=L_{F}(t) 1_{\{t<\zeta\}} d \mathbb{P}_{x}$ on $\mathcal{M}_{t}$. It is known that under these new measures, $X$ is a right process on $E$. We will use $\tilde{X}=\left(\tilde{X}_{t}, \mathcal{M}, \mathcal{M}_{t}, \tilde{\mathbb{P}}_{x}, x \in E\right)$ to denote this new process. Here $\tilde{X}_{t}(\omega)=X_{t}(\omega)$, but we use $\tilde{X}_{t}$ for emphasis when working with $\tilde{\mathbb{P}}_{x}$. This process is called a purely discontinuous Girsanov transform of $X$. 
Remark. The purely discontinuous Girsanov transform was defined for $F \in \mathbf{A}_{2}(X) \subset \mathbf{J}(X) \subset \mathbf{I}_{2}(X)$ (see Ref. 8 for the precise definition). So the purely discontinuous Girsanov transform defined above is more general than that defined in Ref. 8. The stronger assumption $F \in \mathbf{A}_{2}(X)$ is needed in Ref. 8 so one can apply the conditional gauge theorem for nonlocal Feynman-Kac transforms obtained in Refs. 2 and 7 to get estimates on the transition density of the purely discontinuous Girsanov transform of $X$.

First we will determine the Dirichlet form associated with $\tilde{X}$. Define

$$
\tilde{P}_{t} f(x)=\tilde{\mathbb{E}}_{x}\left[f\left(\tilde{X}_{t}\right)\right]=\mathbb{E}_{x}\left[L_{F}(t) f\left(X_{t}\right)\right] .
$$

To prove the next result, we recall the definition of the time-reversal operator $r_{t}$ on the path space. Given a path $\omega \in\{t<\zeta\}$, the operator $r_{t}$ is defined by

$$
r_{t}(\omega)(s)= \begin{cases}\omega(t-s)_{-}, & 0 \leqslant s<t, \\ \omega(0), & s \geqslant t .\end{cases}
$$

Here for $r>0, \omega(r)_{-}:=\lim _{s \uparrow r} \omega(s)$. It is known (see Lemma 4.1.2 of Ref. 12) that the operator $r_{t}$ preserves the measure $\mathbb{P}_{m}$ on $\mathcal{M}_{t} \cap\{t<\zeta\}$.

Lemma 2.1. $\quad \tilde{P}_{t}$ is symmetric on $L^{2}(E, m)$.

Proof. Using the symmetry of $F$, we have

$$
\begin{aligned}
\int_{R^{d}} g(x) \tilde{P}_{t} f(x) d x & =\int_{R^{d}} g(x) \mathbb{E}_{x}\left[\exp \left(M_{F}(t)+\sum_{s \leqslant t}(\ln (1+F)-F)\left(X_{s-}, X_{s}\right)\right) f\left(X_{t}\right)\right] d x \\
& =\mathbb{E}_{m}\left[g\left(X_{0}\right) \exp \left(M_{F}(t)+\sum_{s \leqslant t}(\ln (1+F)-F)\left(X_{s-}, X_{s}\right)\right) f\left(X_{t}\right)\right] \\
& =\mathbb{E}_{m}\left[f\left(X_{0}\right) \exp \left(M_{F}(t)+\sum_{s \leqslant t}(\ln (1+F)-F)\left(X_{s-}, X_{s}\right)\right) \circ r_{t} g\left(X_{t}\right)\right] .
\end{aligned}
$$

From the definition, it is easy to see that $\exp \left(M_{F}(t)+\sum_{s \leqslant t}(\ln (1+F)-\right.$ $\left.F)\left(X_{s-}, X_{s}\right)\right) \circ r_{t}=\exp \left(M_{F}(t)+\sum_{s \leqslant t}(\ln (1+F)-F)\left(X_{s-}, X_{s}\right)\right)$, hence we have shown that

$$
\int_{R^{d}} g(x) \tilde{P}_{t} f(x) d x=\int_{R^{d}} f(x) \tilde{P}_{t} g(x) d x .
$$

Lemma 2.2. Let $A_{t}$ be a positive continuous additive functional of $X$ with Revuz measure $\mu$, then the Revuz measure of $A$ as a positive continuous additive functional of $\tilde{X}$ is also $\mu$. 
Proof. The proof is an easy modification of the proof of Lemma 4.4 of Ref. 11. We omit the details.

Proposition 2.3. Let $f$ be a nonnegative function on $E \times E$ vanishing on the diagonal, we extend it to $E_{\partial} \times E_{\partial}$ by setting it to be zero off $E \times E$. Then for all $t>0$ and $x \in E$ we have

$\tilde{\mathbb{E}}_{x}\left(\sum_{s \leqslant t} f\left(X_{s-}, X_{s}\right)\right)=\tilde{\mathbb{E}}_{x}\left(\int_{0}^{t} \int_{E} f\left(X_{s}, y\right)\left(1+F\left(X_{s}, y\right)\right) N\left(X_{s}, d y\right) d H_{s}\right)$.

Therefore $\left((1+F(x, y)) N(x, d y), H_{t}\right)$ is a Lévy system of $\tilde{X}$.

Proof. Suppose that $f$ is a nonnegative function on $E \times E$ vanishing on the diagonal. For any $n \geqslant 1$, we know that

$$
C_{t}^{n}:=\sum_{s \leqslant t} f\left(X_{s-}, X_{S}\right) 1_{\left\{\left|X_{s-}-X_{s}\right|>\frac{1}{n}\right\}}-\int_{0}^{t} \int_{E_{\partial}} f\left(X_{s}, y\right) 1_{\left\{\left|X_{s}-y\right|>\frac{1}{n}\right\}} N\left(X_{s}, d y\right) d H_{S}
$$

is a $\mathbb{P}_{x}$ martingale for each $x \in E$. It follows from the Girsanov theorem that for any $n \geqslant 1$,

$$
\begin{aligned}
C_{t}^{n}-\int_{0}^{t} \frac{1}{L_{F}(s-)} d\left\langle C^{n}, L^{F}\right\rangle_{s}= & C_{t}^{n}-\left\langle C^{n}, M^{F}\right\rangle_{t} \\
= & \sum_{s \leqslant t} f\left(X_{s-}, X_{s}\right) 1_{\left\{\left|X_{s-}-X_{s}\right|>\frac{1}{n}\right\}} \\
& -\int_{0}^{t} \int_{E} f\left(X_{s}, y\right)\left(1+F\left(X_{s}, y\right)\right) \\
& 1_{\left\{\left|X_{s}-y\right|>\frac{1}{n}\right\}} N\left(X_{s}, d y\right) d H_{s}
\end{aligned}
$$

is a $\tilde{\mathbb{P}}_{x}$ martingale for each $x \in E$, and so

$$
\begin{aligned}
\tilde{\mathbb{E}}_{x}\left(\sum_{s \leqslant t} f\left(X_{s-}, X_{s}\right) 1_{\left\{\left|X_{s-}-X_{s}\right|>\frac{1}{n}\right\}}\right)= & \tilde{\mathbb{E}}_{x}\left(\int_{0}^{t} \int_{E} f\left(X_{s}, y\right)\left(1+F\left(X_{s}, y\right)\right)\right. \\
& \left.1_{\left\{\left|X_{s}-y\right|>\frac{1}{n}\right\}} N\left(X_{s}, d y\right) d H_{s}\right) .
\end{aligned}
$$

Now applying the monotone convergence theorem we get

$$
\tilde{\mathbb{E}}_{x}\left(\sum_{s \leqslant t} f\left(X_{s-}, X_{S}\right)\right)=\tilde{\mathbb{E}}_{x}\left(\int_{0}^{t} \int_{E} f\left(X_{s}, y\right)\left(1+F\left(X_{s}, y\right)\right) N\left(X_{s}, d y\right) d H_{s}\right) .
$$


Let $F_{1}=-F /(1+F)$. By assumption we know that $\inf _{x, y \in E} F(x, y)>$ -1 , so $\inf _{x, y \in E}(1+F(x, y)) \geqslant c$ for some $c \in(0,1)$. Thus $F_{1}^{2} \leqslant F^{2} / c^{2}$, which implies that $F_{1} \in \mathbf{I}_{2}(\tilde{X})$. It is elementary to check that $\inf _{x, y \in E} F_{1}(x, y)>$ -1 . For any $n \geqslant 1$, define $\tilde{M}_{F_{1}}^{(n)}(t)$ to be equal to

$$
\begin{aligned}
& \sum_{s \leqslant t} F_{1}\left(\tilde{X}_{s-}, \tilde{X}_{s}\right) 1_{\left\{\left|\tilde{X}_{s-}-\tilde{X}_{s}\right|>1 / n\right\}}-\int_{0}^{t} \int_{E}\left(F_{1}(1+F)\right) 1_{\left\{\left|\tilde{X}_{s}-y\right|>1 / n\right\}}\left(\tilde{X}_{s}, y\right) N\left(\tilde{X}_{s}, d y\right) d H_{s} \\
& =\sum_{s \leqslant t} F_{1}\left(\tilde{X}_{s-}, \tilde{X}_{s}\right) 1_{\left\{\left|\tilde{X}_{s-}-\tilde{X}_{s}\right|>1 / n\right\}}+\int_{0}^{t} \int_{E} F\left(\tilde{X}_{s}, y\right) 1_{\left\{\left|\tilde{X}_{s}-y\right|>1 / n\right\}} N\left(\tilde{X}_{s}, d y\right) d H_{s} .
\end{aligned}
$$

From Proposition 2.3 we know that $M_{F_{1}}^{(n)}$ is a martingale additive functional of $\tilde{X}$. Let $\tilde{M}_{F_{1}}(t)$ be the limit, as $n \rightarrow \infty$, of $\tilde{M}_{F_{1}}^{(n)}(t)$, in the sense of convergence in the space of square-integrable martingales ( and convergence in probability). Then $\tilde{M}_{F_{1}}(t)$ is a martingale additive functional of $\tilde{X}$. Let $\tilde{L}_{F_{1}}^{(n)}(t)$ be the solution to

$$
\tilde{L}_{F_{1}}^{(n)}(t)=1+\int_{0}^{t} \tilde{L}_{F_{1}}^{(n)}(s-) d \tilde{M}_{F_{1}}^{(n)}(s)
$$

and $\tilde{L}_{F_{1}}(t)$ be the solution to

$$
\tilde{L}_{F_{1}}(t)=1+\int_{0}^{t} \tilde{L}_{F_{1}}(s-) d \tilde{M}_{F_{1}}(s) .
$$

Then by the Doleans-Dade formula it follows that

$$
\begin{aligned}
\tilde{L}_{F_{1}}^{(n)}(t) & \\
= & \exp \left(\tilde{M}_{F_{1}}^{(n)}(t)+\sum_{s \leqslant t}\left(\ln \left(1+F_{1}\right)-F_{1}\right)\left(\tilde{X}_{s-}, \tilde{X}_{s}\right) 1_{\left\{\left|\tilde{X}_{s-}-\tilde{X}_{s}\right|>1 / n\right\}}\right) \\
= & \exp \left(-\sum_{s \leqslant t} \ln (1+F)\left(\tilde{X}_{s-}, \tilde{X}_{s}\right) 1_{\left\{\left|\tilde{X}_{s-}-\tilde{X}_{s}\right|>1 / n\right\}}\right. \\
& \left.+\int_{0}^{t} \int_{E} 1_{\left\{\left|\tilde{X}_{s}-y\right|>1 / n\right\}} F\left(\tilde{X}_{s}, y\right) N\left(\tilde{X}_{s}, d y\right) d H_{s}\right) \\
= & \frac{1}{L_{F}^{(n)}(t)} .
\end{aligned}
$$

In the last equality we used our convention that $\tilde{X}_{t}=X_{t}$. Thus we have shown that

Theorem 2.4. For every $x \in E, \frac{1}{L_{F}(t)}=\tilde{L}_{F_{1}}(t) \tilde{\mathbb{P}}_{x}$-a.s. 
Theorem 2.5. Let $(\tilde{\mathcal{E}}, \tilde{\mathcal{F}})$ be the Dirichlet form of $\tilde{X}$ on $L^{2}(E, m)$. Then $\tilde{\mathcal{F}}=\mathcal{F}$ and for $f \in \mathcal{F}$,

$$
\begin{aligned}
\tilde{\mathcal{E}}(f, f)= & \mathcal{E}^{(c)}(f, f)+\int_{E \times E}(\tilde{f}(x)-\tilde{f}(y))^{2}(1+F)(x, y) J(d x, d y) \\
& +\int_{E} \tilde{f}(x)^{2} \kappa(d x) .
\end{aligned}
$$

Proof. The identification of the Dirichlet form $\tilde{\mathcal{E}}$ on some subset of $\tilde{\mathcal{F}}$ is done in Theorem 4.4 of Ref. 3 under more general assumptions. Here we identify $\tilde{\mathcal{F}}$ with $\mathcal{F}$ and identify $\tilde{\mathcal{E}}$ on all of $\tilde{\mathcal{F}}$. Our proof is similar to that of Theorem 3.4 of Ref. 9. We give the details here for the readers' convenience. It follows from Ref. 16 that there is an $\tilde{\mathcal{E}}$-nest $\left\{K_{n}\right\}$ of compact sets and a sequence $h_{n} \in \mathcal{F}$ such that $\tilde{h}_{n}=1$ on $K_{n}$. Using the probabilistic characterization of nest, we know that $\left\{K_{n}\right\}$ is also an $\mathcal{E}$-nest. For any bounded $f \in \mathcal{F}_{K_{n}}=\left\{g \in \mathcal{F}: \tilde{g}=0\right.$ q. e. on $\left.K_{n}^{c}\right\}$, let $M_{t}^{f}$ be the martinagle part in the Fukushima decomposition of $\tilde{f}\left(X_{t}\right)$. By the Girsanov theorem

$$
K_{t}:=M_{t}^{f}-\int_{0}^{t} \frac{1}{L_{s-}^{F}} d\left\langle M^{f}, L_{F}\right\rangle_{s}=M_{t}^{f}-\left\langle M^{f}, M_{F}\right\rangle_{t}
$$

is a martingale additive functional under $\tilde{\mathbb{P}}_{x}$ and

$$
[K]_{t}\left(\tilde{\mathbb{P}}_{x}\right)=\left[M^{f}\right]_{t}\left(\mathbb{P}_{x}\right), \quad \tilde{\mathbb{P}}_{x}-\text { a.s. }
$$

It follows that

$$
\begin{aligned}
\langle K\rangle_{t}\left(\tilde{\mathbb{P}}_{x}\right) & =\left\langle M^{f}\right\rangle_{t}\left(\mathbb{P}_{x}\right)+\int_{0}^{t} \frac{1}{L_{s-}^{F}} d\left\langle\left[M^{f}\right], L_{F}\right\rangle_{s}\left(\mathbb{P}_{x}\right) \\
& =\left\langle M^{f}\right\rangle_{t}\left(\mathbb{P}_{x}\right)+\left\langle\left[M^{f}\right], M_{F}\right\rangle_{t}\left(\mathbb{P}_{x}\right) \\
& =\left\langle M^{f}\right\rangle_{t}\left(\mathbb{P}_{x}\right)+\int_{0}^{t} \int_{E}\left(\tilde{f}\left(X_{s}\right)-\tilde{f}(y)\right)^{2} F\left(X_{s}, y\right) N\left(X_{s}, d y\right) s H_{s} .
\end{aligned}
$$

Thus by Lemma 2.2 the Revuz measure for the positive continuous additive functional $\langle K\rangle_{t}\left(\tilde{\mathbb{P}}_{x}\right)$ of $\tilde{X}$ is

$$
\begin{aligned}
& \mu_{\langle f\rangle}(d x)+2 \int_{E}(\tilde{f}(x)-\tilde{f}(y))^{2} F(x, y) J(d x, d y) \\
& =\mu_{\langle f\rangle}^{c}(d x)+2 \int_{E}(\tilde{f}(x)-\tilde{f}(y))^{2}(1+F)(x, y) J(d x, d y)+\tilde{f}(x)^{2} \kappa(d x) .
\end{aligned}
$$


Note that $\left\langle M^{f}, M_{F}\right\rangle_{t} \circ r_{t}=\left\langle M^{f}, M_{F}\right\rangle_{t}$ on $\{t<\zeta\}$. So by the Lyons-Zheng forward-backward martingale decomposition we have

$$
\tilde{f}\left(X_{t}\right)-\tilde{f}\left(X_{0}\right)=\frac{1}{2}\left(K_{t}-K_{t} \circ r_{t}\right), \quad \mathbb{P}_{m}-\text { a..s. on }\{t<\zeta\} .
$$

Therefore we have

$$
\begin{aligned}
\lim _{t \downarrow 0} \frac{1}{t} \tilde{\mathbb{E}}_{m}\left[\left(f\left(\tilde{X}_{t}\right)-f\left(\tilde{X}_{0}\right)\right)^{2} ; t<\zeta\right] \\
\leqslant \lim _{t \downarrow 0}\left(\frac{1}{2 t} \tilde{\mathbb{E}}_{m}\left[\left(K_{t}\right)^{2} ; t<\zeta\right]+\frac{1}{2 t} \tilde{\mathbb{E}}_{m}\left[\left(K_{t} \circ r_{t}\right)^{2} ; t<\zeta\right]\right) \\
=\lim _{t \downarrow 0} \frac{1}{t} \mathbb{E}_{m}\left[\langle K\rangle_{t}(\tilde{\mathbb{P}}) ; t<\zeta\right] \\
\leqslant \int_{E} \mu_{\langle f\rangle}^{c}(d x)+2 \int_{E \times E}(\tilde{f}(x)-\tilde{f}(y))^{2}(1+F)(x, y) J(d x, d y) \\
\quad+\int_{E} f(x)^{2} \kappa(d x) \\
\leqslant 2\left(1 \vee\|1+F\|_{\infty}\right) \mathcal{E}(f, f)<\infty .
\end{aligned}
$$

Since $f=0 m$-a.e. on $K_{n}^{c}$ and $h_{n}=1 m$-a.e. on $K_{n}$, we have $f=f h_{n}$ and

$$
\begin{aligned}
& \lim _{t \downarrow 0} \frac{1}{t} \int_{E}\left(f(x)-\tilde{P}_{t} f(x)\right) f(x) m(d x) \\
& =\lim _{t \downarrow 0} \frac{1}{t}\left(\frac{1}{2} \tilde{\mathbb{E}}_{m}\left[\left(f\left(\tilde{X}_{t}\right)-f\left(\tilde{X}_{0}\right)\right)^{2} ; t<\zeta\right]+\int_{E} f(x)^{2}\left(1-\tilde{P}_{t} 1\right)(x) m(d x)\right) \\
& \leqslant \limsup _{t \downarrow 0} \frac{1}{2 t} \tilde{\mathbb{E}}_{m}\left[\left(f\left(\tilde{X}_{t}\right)-f\left(\tilde{X}_{0}\right)\right)^{2} ; t<\zeta\right]+\limsup _{t \downarrow 0} \frac{1}{t} \int_{E}\left(f h_{n}\right)(x)^{2}\left(1-\tilde{P}_{t} 1\right)(x) m(d x) \\
& \leqslant \limsup _{t \downarrow 0} \frac{1}{2 t} \tilde{\mathbb{E}}_{m}\left[\left(f\left(\tilde{X}_{t}\right)-f\left(\tilde{X}_{0}\right)\right)^{2} ; t<\zeta\right] \\
& \quad+\|f\|_{\infty} \limsup _{t \downarrow 0} \frac{1}{t} \int_{E} h_{n}(x)^{2}\left(1-\tilde{P}_{t} 1\right)(x) m(d x) \\
& \leqslant\left(1 \vee\|1+F\|_{\infty}\right) \mathcal{E}(f, f)+\|f\|_{\infty} \tilde{\mathcal{E}}\left(h_{n}, h_{n}\right)<\infty .
\end{aligned}
$$

Therefore $f \in \tilde{\mathcal{F}}$ and so it has a Fukushima decomposition

$$
\tilde{f}\left(\tilde{X}_{t}\right)-\tilde{f}\left(\tilde{X}_{0}\right)=\tilde{M}_{t}^{f}+\tilde{N}_{t}^{f}, \quad \tilde{\mathbb{P}}_{m}-\text { a.s. },
$$

where $\tilde{M}_{t}^{f}$ is a $\tilde{\mathbb{P}}_{x}$-square integrable martingale additive functional of $\tilde{X}$ and $\tilde{N}_{t}^{f}$ is a continuous additive functional of zero energy of $\tilde{X}$. On the other hand, by (2.2) and the Fukushima decomposition

$$
\tilde{f}\left(X_{t}\right)-\tilde{f}\left(X_{0}\right)=M_{t}^{f}+N_{t}^{f},
$$


we have the following decomposition

$$
\tilde{f}\left(X_{t}\right)-\tilde{f}\left(X_{0}\right)=K_{t}+\left(N_{t}^{f}+\left\langle M^{f}, M_{F}\right\rangle_{t}\right) .
$$

Since $N_{t}^{f}+\left\langle M^{f}, M_{F}\right\rangle_{t}$ is a continuous additive functional of zero energy of $\tilde{X}$ and $K_{t}$ is a martingale additive functional of $\tilde{X}$, we have by the uniqueness of the Fukushima decomposition that

$$
\tilde{M}_{t}^{f}=K_{t}, \quad \forall t \geqslant 0 .
$$

Now we are going to find the expression for $\tilde{\mathcal{E}}(f, f)$. The killing measure $\tilde{\kappa}$ of $\tilde{X}$ is the Revuz measure for the positive continuous additive functional $\left(1_{\left\{t \geqslant \zeta_{i}\right\}}\right)^{p}(\tilde{\mathbb{P}})$, the dual predictable projection under $\tilde{\mathbb{P}}$ of the nondecreasing process $t \rightarrow 1_{\left\{t \geqslant \zeta_{i}\right\}}$, where $\zeta_{i}$ is the totally inaccessible part of $\zeta$. Again using the Girsanov theorem, we have

$$
\begin{aligned}
\left(1_{\left\{t \geqslant \zeta_{i}\right\}}\right)^{p}(\tilde{\mathbb{P}})= & \left(1_{\left\{t \geqslant \zeta_{i}\right\}}\right)^{p}(\mathbb{P})+\left\langle 1_{\left\{t \geqslant \zeta_{i}\right\}}, M^{F}\right\rangle_{t}(\mathbb{P}) \\
& =\left(1_{\left\{t \geqslant \zeta_{i}\right\}}\right)^{p}(\mathbb{P})+\left(\left(e^{-F\left(X_{\zeta_{i}}, \partial\right)}-1\right) 1_{\left\{t \geqslant \zeta_{i}\right\}}\right)^{p} \\
& =\left(1_{\left\{t \geqslant \zeta_{i}\right\}}\right)^{p}(\mathbb{P}) .
\end{aligned}
$$

Thus $\tilde{\kappa}(d x)=\kappa(d x)$. And now

$$
\begin{aligned}
\tilde{\mathcal{E}}(f, f) & =\lim _{t \downarrow 0} \frac{1}{2 t} \tilde{\mathbb{E}}_{m}\left[\left(\tilde{f}\left(\tilde{X}_{t}\right)-\tilde{f}\left(\tilde{X}_{0}\right)\right)^{2}, t<\zeta\right]+\int_{E} \tilde{f}(x)^{2} \kappa(d x) \\
& =\lim _{t \downarrow 0} \frac{1}{2 t} \tilde{\mathbb{E}}_{m}\left[\left(\tilde{M}_{t}^{f}\right)^{2}, t<\zeta\right]+\int_{E} \tilde{f}(x)^{2} \kappa(d x) \\
& =\lim _{t \downarrow 0} \frac{1}{2 t} \tilde{\mathbb{E}}_{m}\left[\langle K\rangle_{t}, t<\zeta\right]+\int_{E} \tilde{f}(x)^{2} \kappa(d x) \\
& =\mathcal{E}^{(c)}(f, f)+\int_{E \times E}(\tilde{f}(x)-\tilde{f}(y))^{2}(1+F)(x, y) J(d x, d y)+\int_{E} \tilde{f}(x)^{2} \kappa(d x) .
\end{aligned}
$$

Since $f$ is an arbitrary bounded function in $\mathcal{F}_{K_{n}}$, we conclude that $\cup_{n} \geqslant 1 \mathcal{F}_{K_{n}} \subset \tilde{\mathcal{F}}$ and therefore $\mathcal{F} \subset \tilde{\mathcal{F}}$. Noticing that $F$ and $F_{1}$ play symmetric roles, i. e. $F=-F_{1} /\left(1+F_{1}\right)$, we can interchange the roles of $X$ and $\tilde{X}$ and apply the previous theorem to deduce that $\tilde{\mathcal{F}} \subset \mathcal{F}$ and hence $\mathcal{F}=\tilde{\mathcal{F}}$.

Remark. This theorem is a partial generalization of Theorem 4.8 in Ref. 7, where $F$ was assumed to be in $\mathbf{J}(X)$, while we only assume that $F \in \mathbf{I}_{2}(X)$.

In the remainder of this section we assume that $X$ is a symmetric $\alpha$ stable process in $\mathbb{R}^{d}, \alpha \in(0,2)$. The Dirichlet form corresponding to this process is $(\mathcal{E}, \mathcal{F})$, where 


$$
\begin{aligned}
& \mathcal{E}(u, v)=\frac{1}{2} \mathcal{A}(d,-\alpha) \int_{\mathbb{R}^{d}} \int_{\mathbb{R}^{d}} \frac{(u(x)-u(y))(v(x)-v(y))}{|x-y|^{d+\alpha}} d x d y \\
& \mathcal{F}=\left\{u \in L^{2}\left(\mathbb{R}^{d}\right): \int_{\mathbb{R}^{d}} \int_{\mathbb{R}^{d}} \frac{(u(x)-u(y))^{2}}{|x-y|^{d+\alpha}} d x d y<\infty\right\} .
\end{aligned}
$$

Suppose $F \in \mathbf{I}_{2}(X)$, that is, $F$ is a bounded symmetric function on $\mathbb{R}^{d} \times \mathbb{R}^{d}$ vanishing on diagonal such that for all $x \in \mathbb{R}^{d}$ and $t>0$,

$$
\mathbb{E}_{x}\left[\sum_{s \leqslant t} F^{2}\left(X_{s-}, X_{S}\right)\right]=\mathbb{E}_{x}\left[\int_{0}^{t} \int_{E} \frac{F^{2}\left(X_{s}, y\right)}{\left|X_{S}-y\right|^{d+\alpha}} d y d s\right]<\infty
$$

We are going to establish estimates on the transition density of the pure jump Girsanov $\tilde{X}$ induced by $F$. To do this, we are going to use the following result from Ref. 4.

Theorem 2.6. Suppose that $\alpha \in(0,2)$ and that $c(x, y)$ is a symmetric function on $\mathbb{R}^{d} \times \mathbb{R}^{d}$ such that the function $c(x, y)$ is bounded between two positive numbers $c_{1}$ and $c_{2}$. Then the Markov process corresponding to the Dirichlet form $(\mathcal{Q}, \mathcal{F})$ with

$$
\begin{aligned}
& \mathcal{Q}(u, u)=\int_{\mathbb{R}^{d}} \int_{\mathbb{R}^{d}} \frac{(u(x)-u(y))^{2} c(x, y)}{|x-y|^{d+\alpha}} d x d d y, \quad u \in \mathcal{F} \\
& \mathcal{F}=\left\{u \in L^{2}\left(\mathbb{R}^{d}\right): \int_{\mathbb{R}^{d}} \int_{\mathbb{R}^{d}} \frac{(u(x)-u(y))^{2}}{|x-y|^{d+\alpha}} d x d y<\infty\right\}
\end{aligned}
$$

has a Hölder continuous transition density function $p(t, x, y)$. Furthermore, there are constants $C_{2}>C_{1}>0$ depending only on $c_{1}$ and $c_{2}$ such that

$C_{1}\left(t^{-d / \alpha} \wedge \frac{t}{|x-y|^{d+\alpha}}\right) \leqslant p(t, x, y) \leqslant C_{2}\left(t^{-d / \alpha} \wedge \frac{t}{|x-y|^{d+\alpha}}\right), \quad x, y \in \mathbb{R}^{d}, t>0$.

Proof. It follows from Ref. 5 that the conclusion of the theorem is valid for all $x, y \in \mathbb{R}^{d}$ and $t \in(0,1]$. The conclusion for all $t>0$ follows from an easy scaling argument, like the one used in beginning of the proof of Proposition 4.1 in Ref. 4.

Theorem 2.7. Suppose that $X$ is a symmetric stable process in $\mathbb{R}^{d}$, $\alpha \in(0,2)$, and that $F \in \mathbf{I}_{2}(X)$ satisfies $\inf _{x, y \in \mathbb{R}^{d}} F(x, y)>-1$, then the purely discontinuous Girsanov transform $\tilde{X}$ of $X$ induced by $F$ has a 
Hölder continuous transition density function $p(t, x, y)$ satisfying the estimates

$$
C_{1}\left(t^{-d / \alpha} \wedge \frac{t}{|x-y|^{d+\alpha}}\right) \leqslant p(t, x, y) \leqslant C_{2}\left(t^{-d / \alpha} \wedge \frac{t}{|x-y|^{d+\alpha}}\right), \quad x, y \in \mathbb{R}^{d}, t>0
$$

for some $C_{2}>C_{1}>0$.

Proof. It follows from Theorem 2.5 that the Dirichlet form of $\tilde{X}$ is given by $(\mathcal{Q}, \mathcal{F})$ with

$$
\begin{aligned}
& \mathcal{Q}(u, u)=\int_{\mathbb{R}^{d}} \int_{\mathbb{R}^{d}} \frac{(u(x)-u(y))^{2}(1+F)(x, y)}{|x-y|^{d+\alpha}} d x d d y, \quad u \in \mathcal{F} \\
& \mathcal{F}=\left\{u \in L^{2}\left(\mathbb{R}^{d}\right): \int_{\mathbb{R}^{d}} \int_{\mathbb{R}^{d}} \frac{(u(x)-u(y))^{2}}{|x-y|^{d+\alpha}} d x d d y<\infty\right\} .
\end{aligned}
$$

Now the theorem follows immediately from the result above.

From the theorem above, we immediately get the following

Corollary 2.8. Suppose that $X$ is a symmetric stable process in $\mathbb{R}^{d}$, $\alpha \in(0,2)$, and that $F \in \mathbf{I}_{2}(X)$ satisfies $\inf _{x, y \in \mathbb{R}^{d}} F(x, y)>-1$. If $d>\alpha$, then the purely discontinuous Girsanov transform $\tilde{X}$ of $X$ induced by $F$ has a Green function $G(x, y)$ satisfying

$$
\frac{C_{1}}{|x-y|^{d-\alpha}} \leqslant G(x, y) \leqslant \frac{C_{2}}{|x-y|^{d-\alpha}}, \quad x, y \in \mathbb{R}^{d}
$$

for some $C_{2}>C_{1}>0$.

Remark. Comparing with Theorem 2.2 in Ref. 8, the assumption on the rate at which $F$ tends to zero at the diagonal in the theorem above is much weaker.

\section{Girsanov Transforms of Chen-Zhang}

Suppose that $u$ is a bounded function in $\mathcal{F}_{e}$. Put $\rho(x)=e^{u(x)}$ and $\rho(\partial)=1$. It is easy to check that $\rho-1 \in \mathcal{F}_{e}$. Thus if we define $M^{\rho}:=M^{\rho-1}$ and $N^{\rho}:=N^{\rho-1}$, then we have the Fukushima decomposition for $\tilde{\rho}\left(X_{t}\right)$ :

$$
\tilde{\rho}\left(X_{t}\right)-\tilde{\rho}\left(X_{0}\right)=M_{t}^{\rho}+N_{t}^{\rho} .
$$

Define a square integrable martingale $M$ by

$$
M_{t}=\int_{0}^{t} \frac{1}{\tilde{\rho}\left(X_{s-}\right)} d M_{s}^{\rho} .
$$


Let $L_{t}^{\rho}$ be the solution of the following SDE:

$$
L_{t}^{\rho}=1+\int_{0}^{t} L_{s-}^{\rho} d M_{s} .
$$

It follows from the Doleans-Dade formula that

$$
\begin{aligned}
L_{t}^{\rho} & =\exp \left(M_{t}-\frac{1}{2}\left\langle M^{c}\right\rangle_{t}\right) \prod_{0<s \leqslant t}\left(1+M_{s}-M_{s-}\right) e^{-\left(M_{s}-M_{s-}\right)} \\
& =\exp \left(M_{t}-\frac{1}{2}\left\langle M^{u, c}\right\rangle_{t}\right) \prod_{0<s \leqslant t} \frac{\tilde{\rho}\left(X_{s}\right)}{\tilde{\rho}\left(X_{s-}\right)} \exp \left(1-\frac{\tilde{\rho}\left(X_{s}\right)}{\tilde{\rho}\left(X_{s-}\right)}\right) .
\end{aligned}
$$

$L_{t}^{\rho}$ is a nonnegative local martingale and therefore a supermartingale multiplicative functional of $X$. Therefore by Section 62 of Ref. 17 there is a family of probability measures $\left\{\tilde{\mathbb{P}}_{x}, x \in E\right\}$ on $\mathcal{M}_{\infty}$ such that $d \tilde{\mathbb{P}}_{x}=L_{t}^{\rho} 1_{\{t<\zeta\}} d \mathbb{P}_{x}$ on $\mathcal{M}_{t}$. It is known that under these new measures, $X$ is a right Markov process on $E$. We will use $\tilde{X}=\left(\Omega, \mathcal{M}, \mathcal{M}_{t}, \tilde{X}_{t}, \tilde{\mathbb{P}}_{x}, x \in E\right)$ to denote this new process and it is called the Girsanov transform of $X$ induced by $u$. Here $\tilde{X}_{t}(\omega)=X_{t}(\omega)$ but we use $\tilde{X}_{t}$ for emphasis when working with $\tilde{\mathbb{P}}_{x}$.

The following result is proved in Ref. 9.

Theorem 3.1. $\quad \tilde{X}$ is a symmetric Markov process on $E$ with respect to the measure $\rho^{2} m$ and its Dirichlet form on $L^{2}\left(E, \rho^{2} m\right)$ is $(\tilde{\mathcal{E}}, \mathcal{F})$ with

$$
\begin{aligned}
\tilde{\mathcal{E}}(f, f)= & \frac{1}{2} \int_{E} \tilde{\rho}(x)^{2} \mu_{\langle f\rangle}^{c}(d x)+\int_{E} \tilde{f}(x)^{2} \tilde{\rho}(x) \kappa(d x) \\
& +\int_{E \times E}(\tilde{f}(x)-\tilde{f}(y))^{2} \tilde{\rho}(x) \tilde{\rho}(y) J(d x d y) .
\end{aligned}
$$

In the remainder of this section we assume that $X$ is a symmetric $\alpha$ stable process in $\mathbb{R}^{d}, \alpha \in(0,2]$. When $\alpha \in(0,2)$, the Dirichlet form corresponding to this process is $(\mathcal{E}, \mathcal{F})$, where

$$
\begin{aligned}
& \mathcal{E}(u, v)=\frac{1}{2} \mathcal{A}(d,-\alpha) \int_{\mathbb{R}^{d}} \int_{\mathbb{R}^{d}} \frac{(u(x)-u(y))(v(x)-v(y))}{|x-y|^{d+\alpha}} d x d y \\
& \mathcal{F}=\left\{u \in L^{2}\left(\mathbb{R}^{d}\right): \int_{\mathbb{R}^{d}} \int_{\mathbb{R}^{d}} \frac{(u(x)-u(y))^{2}}{|x-y|^{d+\alpha}} d x d y<\infty\right\} .
\end{aligned}
$$

When $\alpha=2$, the Dirichlet form corresponding to this process is $(\mathcal{E}, \mathcal{F})$, where $\mathcal{F}=H^{1}\left(\mathbb{R}^{d}\right)$ and

$$
\mathcal{E}(u, v)=\int_{\mathbb{R}^{d}} \nabla u(x) \cdot \nabla v(x) d x .
$$


Theorem 3.2. Suppose that $X$ is a symmetric $\alpha$-stable process in $\mathbb{R}^{d}$, $\alpha \in(0,2]$. When $u$ is a bounded function in $\mathcal{F}_{e}$, the Girsanov transform $\tilde{X}$ of $X$ induced by $u$ has a Hölder continuous transition density $p(t, x, y)$ with respect to Lebesgue measure on $\mathbb{R}^{d}$. When $\alpha \in(0,2), p(t, x, y)$ satisfies

$$
C_{1}\left(t^{-d / \alpha} \wedge \frac{t}{|x-y|^{d+\alpha}}\right) \leqslant p(t, x, y) \leqslant C_{2}\left(t^{-d / \alpha} \wedge \frac{t}{|x-y|^{d+\alpha}}\right), \quad x, y \in \mathbb{R}^{d}, t>0
$$

for some $C_{2}>C_{1}>0$; while when $\alpha=2, p(t, x, y)$ satisfies the following Gaussian estimates

$$
C_{3} t^{-d / 2} \exp \left(-\frac{C_{4}|x-y|^{2}}{t}\right) \leqslant p(t, x, y) \leqslant C_{5} t^{-d / 2} \exp \left(-\frac{C_{6}|x-y|^{2}}{t}\right)
$$

for some positive constants $C_{3}, C_{4}, C_{5}, C_{6}$.

Proof. When $\alpha \in(0,2)$, by Theorem 3.1 we know that the Dirichlet form on $L^{2}\left(\mathbb{R}^{d}, \rho^{2} d x\right)$ is $(\tilde{\mathcal{E}}, \tilde{\mathcal{F}})$, where $\tilde{\mathcal{F}}=\mathcal{F}$ and

$$
\tilde{\mathcal{E}}(f, f)=\frac{1}{2} \mathcal{A}(d,-\alpha) \int_{\mathbb{R}^{d}} \int_{\mathbb{R}^{d}} \frac{(f(x)-f(y))^{2} e^{u(x)} e^{u(y)}}{|x-y|^{d+\alpha}} d x d y .
$$

Now the conclusion of the theorem follows immediately from Theorem 2.6.

When $\alpha=2$, by Theorem 3.1 we know that the Dirichlet form on $L^{2}\left(\mathbb{R}^{d}, \rho^{2} d x\right)$ is $(\tilde{\mathcal{E}}, \tilde{\mathcal{F}})$, where $\tilde{\mathcal{F}}=\mathcal{F}$ and

$$
\tilde{\mathcal{E}}(f, f)=\int_{\mathbb{R}^{d}}|\nabla f(x)|^{2} e^{2 u(x)} d x .
$$

Now our theorem follows immediately from the famous Aronson estimates.

Corollary 3.3. Let $\alpha \in(0,2]$ and $d>\alpha$. Suppose that $X$ is a symmetric $\alpha$-stable process in $\mathbb{R}^{d}$. If $u$ is a bounded function in $\mathcal{F}_{e}$, then the Girsanov transform $\tilde{X}$ induced by $u$ has a Green function $G(x, y)$ satisfying

$$
\frac{C_{1}}{|x-y|^{d-\alpha}} \leqslant G(x, y) \leqslant \frac{C_{2}}{|x-y|^{d-\alpha}}
$$

for some $C_{2}>C_{1}>0$. 
Now we apply the results above to get estimates on the density, when it exists, of the potential operator of the Feynman-Kac semigroup

$$
T_{t} f(x)=\mathbb{E}_{x}\left[\exp \left(N_{t}^{u}\right) f\left(X_{t}\right)\right],
$$

where $N^{u}$ is continuous additive functional of zero energy in the Fukushima decomposition of $\tilde{u}\left(X_{t}\right)$. This semigroup was first studied in Refs. 13 and 14 .

Recall that for a transient symmetric Markov process on $\mathbb{R}^{d}$ with Green function $G(x, y)$, a Radon measure $\mu$ is said to be in the Kato class $\mathbf{K}_{\infty}(X)$ if

$$
\lim _{r \downarrow 0} \sup _{x \in \mathbb{R}^{d}} \int_{B(x, r)} G(x, y)|\mu|(d y)=0
$$

and

$$
\lim _{R \uparrow \infty} \int_{B(x, R)^{c}} G(x, y)|\mu|(d y)=0 .
$$

Theorem 3.4. Let $\alpha \in(0,2]$ and $d>\alpha$. Suppose that $X$ is a symmetric $\alpha$-stable process in $\mathbb{R}^{d}$, and that $u$ is a bounded function in $\mathcal{F}_{e}$ with $\mu_{<u>} \in \mathbf{K}_{\infty}(X)$. If for some $x \in \mathbb{R}^{d}, \int_{0}^{\infty} T_{t} 1(x) d t<\infty$, then the potential operator $\int_{0}^{\infty} T_{t} d t$ of the semigroup $\left(T_{t}\right)$ has a density $G(x, y)$ with respect to the Lebesgue measure satisfying

$$
\frac{C_{1}}{|x-y|^{d-\alpha}} \leqslant G(x, y) \leqslant \frac{C_{2}}{|x-y|^{d-\alpha}}
$$

for some $C_{2}>C_{1}>0$.

Proof. We only give the proof in the case of $\alpha \in(0,2)$, the proof in the case of $\alpha=2$ is similar. By (3.6) of Ref. 9,

$$
\begin{aligned}
T_{t} f(x) & =\mathbb{E}_{x}\left[f\left(X_{t}\right) e^{\tilde{u}\left(X_{t}\right)-\tilde{u}\left(X_{0}\right)-M_{t}^{u}}\right] \\
& =e^{-u(x)} \mathbb{E}_{x}\left[L_{t}^{e^{-u}} e^{-A_{t}^{-u}}\left(f e^{u}\right)\left(X_{t}\right)\right] \\
& =e^{-u(x)} \tilde{\mathbb{E}}_{x}\left[e^{-A_{t}^{-u}}\left(f e^{u}\right)\left(\tilde{X}_{t}\right)\right]
\end{aligned}
$$

where $\tilde{X}$ is the Girsanov transform of $X$ induced by $-u$ and

$$
A_{t}^{-u}=\mathcal{A}(d,-\alpha) \int_{0}^{t} \int_{\mathbb{R}^{d}}\left(\tilde{u}\left(X_{s}\right)-\tilde{u}(y)+1-e^{\tilde{u}\left(X_{s}\right)-\tilde{u}(y)}\right) \frac{1}{\left|X_{s}-y\right|^{d+\alpha}} d y d s .
$$

The Revuz measure for $A^{-u}$ as an additive functional of $X$ is $\mu(d x)=2 \mathcal{A}(d,-\alpha) \int_{\mathbb{R}^{d}}\left(\tilde{u}(x)-\tilde{u}(y)+1-e^{\tilde{u}(x)-\tilde{u}(y)}\right) \frac{1}{\left|X_{S}-y\right|^{d+\alpha}} d y \leqslant c \mu_{<u>}(d x)$ 
for some constant $c>0$. By Lemma 4.2 of Ref. 9, the Revuz measure for $A^{-u}$ as an additive functional of $X$, is $e^{-2 u} \mu$. Therefore by Corollary 3.3 we know that $e^{-2 u} \mu \in \mathbf{K}_{\infty}(\tilde{X})$. If for some $x \in \mathbb{R}^{d}, \int_{0}^{\infty} T_{t} 1(x) d t<\infty$, then by (3.1) we have $\int_{0}^{\infty} \tilde{\mathbb{E}}_{x}\left[e^{-A_{t}^{-u}}\right] d t<\infty$. Now applying Theorem 3.6 of Ref. 2 we see that $\left(\tilde{X}, e^{-2 u} \mu\right)$ is conditionally gaugeable. Therefore by Lemma 3.5 of Ref. 2 and Theorem 32 we get that the operator

$$
\tilde{G} f(x)=\int_{0}^{\infty} \tilde{\mathbb{E}}_{x}\left[e^{-A_{t}^{-u}} f\left(\tilde{X}_{t}\right)\right] d t, \quad x \in \mathbb{R}^{d}
$$

has a density $\tilde{G}(x, y)$ with respect to the measure $e^{-2 u(x)} d x$ such that

$$
\frac{c_{1}}{|x-y|^{d-\alpha}} \leqslant \tilde{G}(x, y) \leqslant \frac{c_{2}}{|x-y|^{d-\alpha}}
$$

for some $c_{2}>c_{1}>0$. Using (3.1) we see that the potential operator $\int_{0}^{\infty} T_{t} d t$ of the semigroup $\left(T_{t}\right)$ has a density $G(x, y)$ with respect to the Lebesgue measure given by

$$
G(x, y)=\frac{\tilde{G}(x, y)}{e^{u(x)} e^{u(y)}}, \quad x, y \in \mathbb{R}^{d} .
$$

Now the conclusion of the theorem follows easily.

Remark. The conclusion of the theorem above is connected with the conditional gauge theorems proved in Refs. 2, 6, and 7. One of the consequences of the conditional gauge theorem is the comparability of Green functions. However, as far as I know, there is no satisfactory conditional gauge theorem for continuous additive functionals of zero energy yet.

\section{ACKNOWLEDGMENT}

I thank Zhen-Qing Chen and Zoran Vondracek for their comments on the first version of this paper. I also thank the referee for very helpful comments.

\section{REFERENCES}

1. Benveniste, A., and Jacod, J. (1973). Systèmes de Lévy des processus de Markov. Invent. Math. 21, 183-198.

2. Chen, Z.-Q. (2002). Gaugeability and conditional gaugeability. Trans. Amer. Math. Soc. 354, 4639-4679.

3. Chen, Z.-Q., Fitzsimmons, P. J., Takeda, M., Ying, J., and Zhang, T.-S. (2004). Absolute continuity of symmetric Markov processes. Ann. Probab. 32, 2067-2098.

4. Chen, Z.-Q., and Kumagai, T. (2003). Heat kernel estimates for stable-like processes on $d$-sets. Stochastic Process. Appl. 108, 27-62. 
5. Chen, Z.-Q., Ma, Z., and Röckner, M. (1994). Quasi-homeomorphisms of Dirichlet forms. Nagoya Math. J. 136, 1-15.

6. Chen, Z.-Q., and Song, R. (2002). General gauge and conditional gauge theorems. Ann. Probab. 30, 1313-1339.

7. Chen, Z.-Q., and Song, R. (2003). Conditional gauge theorem for non-local Feynman-Kac transforms. Probab. Theo. relat. Fields 125, 45-72.

8. Chen, Z.-Q., and Song, R. (2003). Drift transforms and Green function estimates for discontinuous processes. J. Funct. Anal. 201, 262-281.

9. Chen, Z.-Q., and Zhang, T.-S. (2002). Girsanov and Feynman-Kac type transformations for symmetric Markov processes. Ann. Inst. Henri Poincaré 38, 475-505.

10. Cranston, M., and Zhao, Z. (1987). Conditional transformation of drift formula and potential theory for $\frac{1}{2} \Delta+b(\cdot) \cdot \nabla$. Commun. Math. Phys. 112, 613-625.

11. Fitzsimmons, P. J. (1997). Absolute continuity of symmetric diffusions. Ann. Probab. 25, 230-258.

12. Fukushima, M., Oshima, Y., and Takeda, M. (1994). Dirichlet forms and symmetric Markov processes, Walter De Gruyter, Berlin.

13. Glover, J., Rao, M., and Song, R. (1993). Generalized Schrödinger semigroups. In Cinlar, E., Chung, K. L., and Getoor, R. K. (eds.), Seminar on Stochastic Processes, 1992, Birhaüser, Boston.

14. Glover, J., Rao, M., Sikic, H., and Song, R. (1994). Quadratic forms corresponding to the generalized Schrödinger semigroups. J. Funct. Anal. 125, 358-378.

15. He, S. W., Wang, J. G., and Yan, J. A. (1992). Semimartingale Theory and Stochastic Calculus, Science Press, Beijing New York.

16. Ma, Z., and Röckner, M. (1992). Introduction to the Theory of (Non-symmetric) Dirichlet Forms, Springer, Berlin.

17. Sharpe, M. (1988). General Theory of Markov Processes, Academic Press, Boston. 